\title{
Overexpression of long non-coding RNA H19 is associated with unfavorable prognosis in patients with colorectal cancer and increased proliferation and migration in colon cancer cells
}

\author{
SHAN-WEN CHEN, JING ZHU, JU MA，JUN-LING ZHANG，SHUAI ZUO，GUO-WEI CHEN, \\ XIN WANG, YI-SHENG PAN, YU-CUN LIU and PENG-YUAN WANG
}

Division of General Surgery, Peking University First Hospital, Peking University, Beijing 100034, P.R. China

Received December 11, 2015; Accepted April 21, 2017

DOI: $10.3892 / 01.2017 .6390$

\begin{abstract}
Long non-coding RNA-imprinted maternally expressed transcript (non-protein coding) (H19) has been previously identified to be involved in the development of a number of types of cancer. However, the function of H19 in the pathogenesis of colorectal cancer remains unclear. The expression level of H19 in colorectal tumor tissues, and the association between H19 expression and clinicopathological variables and prognosis was investigated in the present study. In addition, the effect of H19 overexpression on viability, migration and epithelial-mesenchymal transition (EMT) of colon cancer cells was investigated in HCT-116 and SW-480 cells. The results of the present study suggest that overexpression of H19 is associated with decreased recurrence-free survival and overall survival rates in patients with colorectal cancer, and increased viability and migration in colon cancer cells. The induction of the EMT process may be an underlying molecular mechanism associated with the H19-induced increased metastasis potential of colon cancer cells.
\end{abstract}

\section{Introduction}

Colorectal cancer is one of the most common types of cancer in males and females. It is estimated that there are $\sim 140,000$ novel cases of colorectal cancer expected in the USA and it remains the third leading cause of cancer-associated mortality in 2014 (1). In China, colorectal cancer was the fifth most common type of cancer and the fifth leading cause of cancer-associated mortality in 2011 (2). Although the 5-year mortality rate of colorectal cancer has slightly decreased in the last decade, novel prognostic factors and potential therapeutic

Correspondence to: Professor Peng-Yuan Wang, Division of General Surgery, Peking University First Hospital, Peking University, 8 Xi Shiku Street, Beijing 100034, P.R. China

E-mail: wangpengyuan2014@126.com

Key words: colorectal cancer, H19, metastasis, overall survival, recurrence-free survival, epithelial-mesenchymal transition targets for this disease are required. Furthermore, the underlying pathophysiological mechanisms of the development of colorectal cancer remain unclear $(3,4)$.

Long non-coding RNAs (lncRNAs), >200 nucleotides in length, have been identified as novel gene expression regulators in the last decade $(5,6)$. A previous study suggested that a number of lncRNAs may be involved in the development and metastasis of cancer (7). LncRNA-imprinted maternally expressed transcript (non-protein coding) (H19), initially reported in 1991 by Bartolomei et al (8), was identified to be expressed at increased levels in extraembryonic tissues, embryonic tissues and the majority of fetal tissues; however, the expression level of lncRNA H19 is markedly decreased following birth (9). As the first imprinting lncRNA to be identified, H19, which is expressed by the maternal allele rather than the paternal, is transcribed from the H19/insulin-like growth factor 2 gene cluster located on human chromosome 11 15.5 (10). Previous studies have revealed that H19 serves an important function in the progression of a number of types of cancer (11-13). Additionally, H19 overexpression was identified to be markedly associated with a poor prognosis in bladder and gastric cancer $(14,15)$. Currently, the expression levels and the function of H19 in colorectal cancer remain unclear.

In the present study, the expression level of H19 and its association with clinicopathological variables and disease outcome was examined using colorectal tumor samples and paired adjacent normal tissues. In addition, the effects of H19 overexpression on the viability, migration and EMT process of colon cancer cells were investigated in HCT-116 and SW-480 cells, using an H19-expressing plasmid. The results suggested that H19 overexpression was significantly associated with pre-treatment metastasis $(\mathrm{P}=0.020)$, poor differentiation level $(\mathrm{P}=0.022)$ and advanced tumor-node-metastasis (TNM) stages $(\mathrm{P}=0.046)$. Furthermore, H19 overexpression was identified to be an independent risk factor for decreased recurrence-free survival (RFS) [hazard ratio (HR), 3.037; 95\% confidence interval (CI), 1.107-8.329; $\mathrm{P}=0.031$ ] and overall survival (OS) time (HR, 4.028; 95\% CI, 1.332-12.183; $\mathrm{P}=0.014)$ in patients with colorectal cancer. H19 overexpression was identified to be associated with increased viability and migratory potential of HCT-116 and SW-480 cells. Additionally, H19 overexpression decreased the expression level of epithelial (E-)cadherin and 
increased the expression levels of vimentin and snail family transcriptional repressor 1 (Snail) in colon cancer cells. Immunofluorescence suggested that H19 overexpression increased the expression of filamentous (F-) actin in SW-480 cells. The results of the present study suggested that H19 overexpression is associated with decreased RFS and OS rates in patients with colorectal cancer, and increased viability and migration of colon cancer cells.

\section{Materials and methods}

Patients and samples. Between April 2006 and October 2009, 96 patients with colorectal cancer, who underwent surgery at Peking University First Hospital (Beijing, China), were included in the present study. Baseline characteristics of all patients, including sex, age, tumor size, tumor histological differentiation status, the status of mucin and TNM stages, according to the American Joint Committee on Cancer staging (7th edition), were collected from the medical records and surgical pathology reports (Table I) (16). Patient follow-up was carried out every 3 months for the first 2 years, every 6 months for the following 3 years and annually thereafter. The present study was approved by the Institutional Review Board at Peking University First Hospital, conducted in accordance with the principles of The Declaration of Helsinki and written informed consent was obtained from all patients. Samples of colorectal tumors and paired adjacent normal tissues were collected immediately following resection, and stored at $-80^{\circ} \mathrm{C}$ until use.

Reverse transcription-quantitative polymerase chain reaction (RT-qPCR) to detect H19. Total RNA was extracted using the TRIzol one-step extraction method (TRIzol reagent; Invitrogen; Thermo Fisher Scientific, Inc., Waltham, MA, USA) and reverse-transcribed into cDNA using a Reverse Transcription System kit (cat. no. A3500; Promega Corporation, Madison, WI, USA), according to the manufacturer's protocol. qPCR analysis was carried out using a final reaction volume of $25 \mu \mathrm{l}$ and the TaqMan ${ }^{\circledR}$ Universal PCR Master Mix (Applied Biosystems; Thermo Fisher Scientific, Inc.), according to the manufacturer's protocol. All reactions were performed in triplicate using a 7500 Real-Time PCR System (Applied Biosystems; Thermo Fisher Scientific, Inc.). The thermocycling conditions comprised the following steps: initial denaturation for $15 \mathrm{~min}$ at $95^{\circ} \mathrm{C}$, then 40 cycles of denaturation at $94^{\circ} \mathrm{C}$ for $15 \mathrm{sec}$, then annealing for $30 \mathrm{sec}$ at $55^{\circ} \mathrm{C}$ and extension for $30 \mathrm{sec}$ at $72^{\circ} \mathrm{C}$. Relative RNA expression was calculated as a fold-change using the comparative quantification $(\mathrm{Cq})$ method $\left(2^{-\Delta \Delta \mathrm{Cq}}\right)$, with GAPDH as the internal control gene (17). The primers and probes used, listed 5'-3', were as follows: GAPDH forward, CAGTCAGCCGCATCTTCTTTT; GAPDH reverse, GTGACCAGGCGCCCAATAC; GAPDH probe, tetramethylrhodamine (TAMRA)-CGTCGCCAGCCG AGCCACA-Black Hole Quencher (BHQ)2; H19 forward, AATCGGCTCTGGAAGGTGAA; H19 reverse, CTGCTG TTCCGATGGTGTCTT; H19 probe, TAMRA-CTAGAG GAACCAGACCTCATCAGCCCAAC-BHQ1.

Cell culture. Human colon cancer cell lines HCT-116 and SW-480 were purchased from the American Type Culture Collection (Manassas, VA, USA). HCT-116 cells were
Table I. Association between H19 expression and clinicopathological variables.

\begin{tabular}{|c|c|c|c|c|}
\hline \multirow[b]{2}{*}{ Characteristic } & \multirow{2}{*}{$\frac{\text { Total, } \mathrm{n}=96}{\text { No. }}$} & \multicolumn{2}{|c|}{$\begin{array}{c}\text { H19 } \\
\text { expression }\end{array}$} & \multirow[b]{2}{*}{ P-value } \\
\hline & & High & Low & \\
\hline Sex & & & & 0.365 \\
\hline Male & 42 & 21 & 21 & \\
\hline Female & 54 & 32 & 22 & \\
\hline Age, years & & & & 0.845 \\
\hline$\leq 60$ & 39 & 22 & 17 & \\
\hline$>60$ & 57 & 31 & 26 & \\
\hline T-stage & & & & 0.646 \\
\hline $\mathrm{T} 1-2$ & 16 & 8 & 8 & \\
\hline T3-4 & 80 & 45 & 35 & \\
\hline N-stage & & & & 0.874 \\
\hline N0-1 & 84 & 45 & 36 & \\
\hline N2 & 12 & 8 & 7 & \\
\hline M-stage & & & & 0.020 \\
\hline M0 & 77 & 38 & 39 & \\
\hline M1 & 19 & 15 & 4 & \\
\hline \multicolumn{5}{|l|}{ Tumor size, $\mathrm{cm}$} \\
\hline$\leq 5$ & 75 & 40 & 35 & 0.485 \\
\hline$>5$ & 21 & 13 & 8 & \\
\hline Differentiation & & & & 0.022 \\
\hline Well/moderate & 83 & 42 & 41 & \\
\hline Poor & 13 & 11 & 2 & \\
\hline Mucin production & & & & 0.315 \\
\hline Absent & 82 & 47 & 35 & \\
\hline Present & 14 & 6 & 8 & \\
\hline TNM stage & & & & 0.046 \\
\hline I-II & 54 & 25 & 29 & \\
\hline III-IV & 42 & 28 & 14 & \\
\hline
\end{tabular}

P-values in italic indicate statistically significant differences. H19, long non-coding RNA-imprinted maternally expressed transcript; TNM, tumor-node-metastasis.

cultured in McCoy's 5A medium (Thermo Fisher Scientific, Inc.) with $10 \%$ fetal bovine serum (FBS; Thermo Fisher Scientific, Inc.). SW-480 cells were cultured in Dulbecco's modified Eagle's medium (DMEM; Thermo Fisher Scientific, Inc.) with $10 \%$ FBS. H19 expressing plasmid pcDNA 3.1 (+)-H19 (cat. no. C05003) or pcDNA 3.1(+) empty vector as control were purchased from Suzhou GenePharma Co., Ltd. (Suzhou, China). Transfection was conducted using Lipofectamine ${ }^{\circledR} 3000$ (Thermo Fisher Scientific, Inc.), according to the manufacturer's protocol. A total of $1 \times 10^{6}$ cells were seeded for transfection.

MTT viability assays. A total of 1,000 cells/well were seeded in a 96-well plate for $24 \mathrm{~h}$. The cells were subsequently transfected with $0.2 \mu \mathrm{g} \mathrm{H} 19$ or control plasmid for $24 \mathrm{~h}$ and 


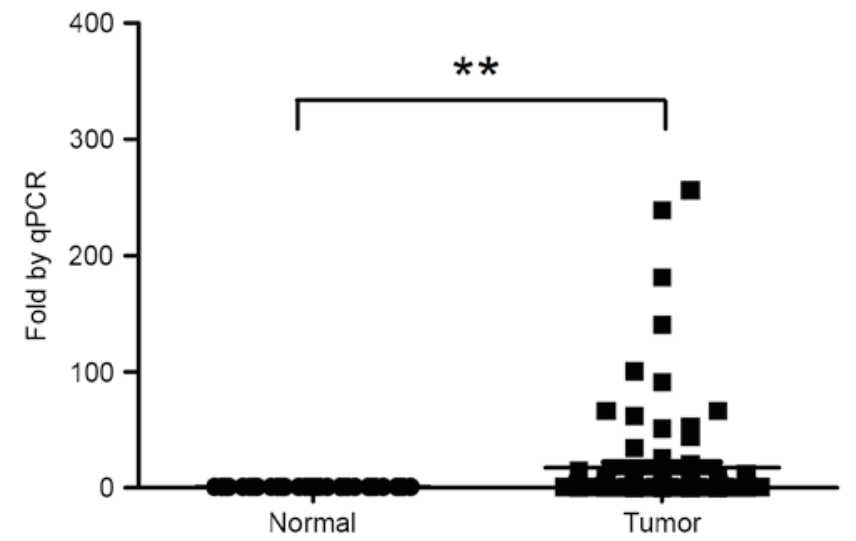

Figure 1. H19 expression in rectal tumor tissues is significantly increased, compared with adjacent normal tissues. The means of the two groups were compared using Wilcoxon signed-rank test $\left({ }^{* *} \mathrm{P}<0.01\right.$ vs. normal) qPCR, quantitative polymerase chain reaction; H19, long non-coding RNA-imprinted maternally expressed transcript.

incubated at $37^{\circ} \mathrm{C}$ for 5 days. The cells were incubated in $50 \mu \mathrm{l}$ $0.1 \mathrm{mg} / \mathrm{ml}$ solution of MTT (Sigma-Aldrich, USA) at $37^{\circ} \mathrm{C}$ for $4 \mathrm{~h}$ and subsequently lysed in $150 \mu \mathrm{l}$ dimethyl sulfoxide at room temperature for $30 \mathrm{~min}$. The absorbance in each well was measured at $580 \mathrm{~nm}$ using a microplate reader. Experiments were performed in triplicate and repeated 3 times.

Migration assays. Cell migration assays were analyzed using Transwell chambers $(8.0 \mu \mathrm{m}$ pore size; Corning Incorporated, Corning, NY, USA). At 24 h post-transfection, with H19 or control plasmid, cells were incubated in serum-free medium for $12 \mathrm{~h}$ at $37^{\circ} \mathrm{C}$. A total of $5 \times 10^{4}$ cells, in $200 \mu \mathrm{l}$ DMEM or MacCoy's 5A medium without serum, were added to the upper chamber and $600 \mu \mathrm{l}$ DMEM or MacCoy's 5A medium with $20 \%$ FBS was placed in the bottom of wells. The plates were incubated at $37^{\circ} \mathrm{C}$ in an atmosphere containing $5 \% \mathrm{CO}_{2}$ for $24 \mathrm{~h}$. The cells that migrated to the opposite side of the membrane were fixed and stained with hematoxylin and eosin, and the number of invading cells was determined using a microscope (Olympus Corporation, Tokyo, Japan) using a previously described method (18). Experiments were performed in triplicate and repeated 3 times.

Western blot analysis. Total protein was extracted from cells $72 \mathrm{~h}$ after transfection with $\mathrm{H} 19$ or control plasmid using a Cell Total Protein Extraction kit, (Beyotime Institute of Biotechnology, Haimen, China). The concentrations of protein were determined using the bicinchoninic acid assay method and equal quantities of $25 \mu \mathrm{g}$ protein/lane were separated by SDS-PAGE (10\% gel). Subsequently, the separated proteins were transferred onto a polyvinylidene fluoride membrane. The membrane was blocked for non-specific binding for $1 \mathrm{~h}$ [5\% bovine serum albumin (BSA) in TBS-Tween-20 buffer] at room temperature, and subsequently incubated overnight at $4^{\circ} \mathrm{C}$ with the following rabbit monoclonal antibodies: anti-E-cadherin (cat. no. 3195; dilution, 1:1,000; Cell Signaling Technology, Inc., Danvers, MA, USA), rabbit anti-vimentin (cat. no. 5741; dilution, 1:1,000), rabbit anti-Snail (cat. no. 3879; dilution, 1:1,000) and rabbit anti-GAPDH (cat. no. 2118; dilution, 1:1,000) (all from Cell Signaling Technology, Inc.).
The membrane was subsequently incubated at room temperature for $1 \mathrm{~h}$ with anti-rabbit immunoglobulin $\mathrm{G}$ horseradish peroxidase-linked secondary antibodies (cat. no. 7074; dilution, 1:5,000; Cell Signaling Technology, Inc.). Blots were developed with enhanced chemiluminescence detection reagents (Merck KGaA, Darmstadt, Germany).

Immunofluorescence. The immunofluorescence of F-actin was carried out according to a previously described protocol (19), with certain modifications. Following transfection with H19 or control plasmid for 72 h, SW-480 cells, on glass slides, were fixed with ice-cold acetone for $5 \mathrm{~min}$ at $-20^{\circ} \mathrm{C}$. Subsequently, slides were placed at the bottom of the wells of the 6-well plates with the cells facing upwards. Slides were rinsed with PBS, followed by blocking with $1 \%$ BSA for $1 \mathrm{~h}$ at room temperature. F-actin was stained following incubation with rhodamine-phalloidin (Thermo Fisher Scientific, Inc.) and nuclei were stained using DAPI (Thermo Fisher Scientific, Inc.). Following washing with PBS, slides were mounted, using the ProLong Gold Antifade reagent (Molecular Probes, USA), and stored at $4^{\circ} \mathrm{C}$ in the dark until analyzed. Fluorescence was visualized using a Fluoview 1000 confocal microscope (Olympus Corporation).

Statistical analysis. The results for each tumor were evaluated by determining the relative ratio of tumor to normal tissue in the same patient. Data were tested for normal distribution using the Kolmogorov-Smirnov test and the means of the two groups were compared using Wilcoxon signed-rank test. A relative ratio of tumor to normal tissue $\geq 4$ and $<4$ was classified as high expression and low expression, respectively. Pearson's $\chi^{2}$ test was used to analyze the association between H19 expression and clinicopathological variables. Kaplan-Meier estimator and Cox's proportional hazard tests were used for survival analysis. The results were expressed as the mean \pm standard error of the mean and analyzed using a Student's t-test for unpaired data. $\mathrm{P}<0.05$ indicated a statistically significant difference. All statistical analyses were carried out using SPSS software (version 23.0; IBM Corp., Armonk, NY, USA).

\section{Results}

Patient characteristics. The male to female ratio of the study population was $0.78: 1$ and the median age was 65 years (range, $25-84$ years). A total of 40 patients (42\%) exhibited lymphoid node metastasis and 19 patients $(20 \%)$ exhibited distant metastasis at the time of surgery. The mean follow-up period was 45 months (range, 3-60 months). In total, 34 patients (35\%) experienced tumor recurrence and distant recurrence occurred in 15 patients (16\%; Table I).

Relative H19 expression in tumor and paired adjacent normal tissues. The Kolmogorov-Smirnov test indicated that the expression of H19 in tumor tissues exhibited a non-normal distribution $(\mathrm{P}<0.001)$. Analysis of 96 paired rectal tumors and paired adjacent normal tissues revealed a significant upregulation (median, 4.19-fold) of H19 expression in tumor tissues compared with matched normal tissues (Fig. 1). The 96 patients were subsequently divided into two groups on the basis of the fold change of H19 expression [H19-high ( $\geq 4$-fold 
A

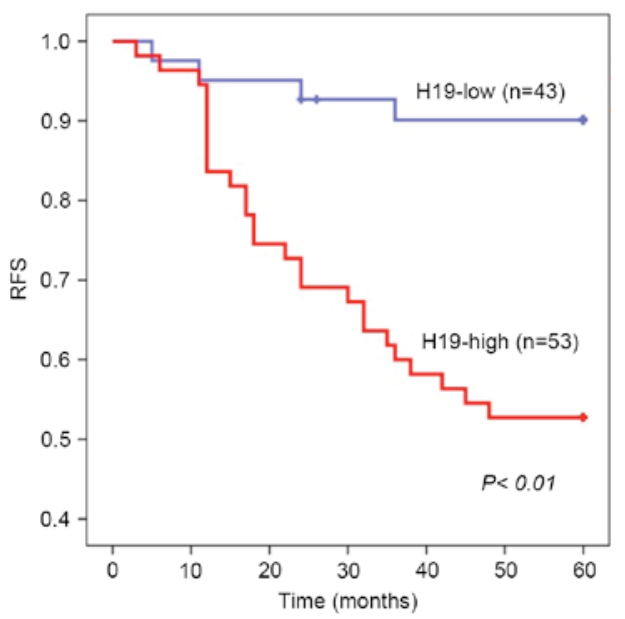

B

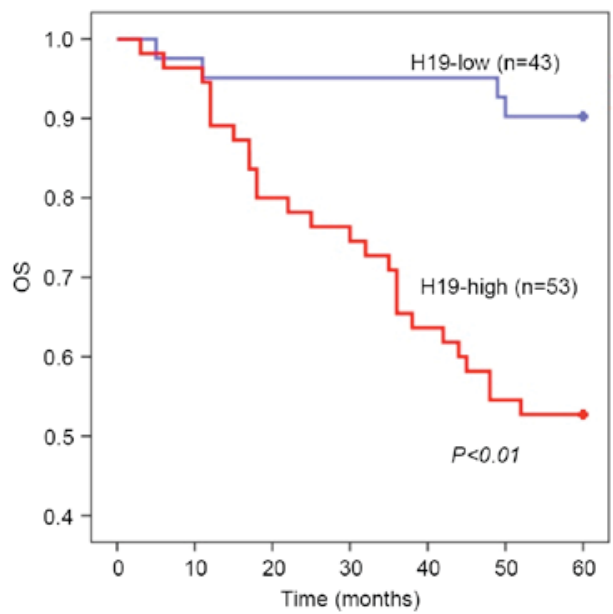

Figure 2. Kaplan-Meier estimator analysis of (A) RFS and (B) OS rates, according to H19 expression. Statistical analysis by log-rank test, P<0.01. RFS, recurrence-free survival; OS, overall survival; H19, long non-coding RNA-imprinted maternally expressed transcript.

upregulation, $\mathrm{n}=53)$ and $\mathrm{H} 19$-low (<4-fold upregulation, $\mathrm{n}=43)]$.

Association between $H 19$ expression with clinicopathological variables. Table I demonstrates the association between H19 expression in colorectal tumor tissues and clinicopathological variables. H19 overexpression in colorectal tumor tissues was identified to be associated with pre-treatment metastasis $(\mathrm{P}=0.020)$, poor differentiation level $(\mathrm{P}=0.022)$ and advanced TNM stages $(\mathrm{P}=0.046)$.

Expression of $\mathrm{H} 19$ in association with prognosis of rectal cancer. Kaplan-Meier estimator survival analysis identified that overexpression of $\mathrm{H} 19$ in colorectal tumor tissues was significantly associated with poor RFS $(\mathrm{P}<0.01)$ and OS $(\mathrm{P}<0.01)$ rates (Fig. 2A and B). Cox's univariate proportional hazard analysis of RFS revealed that lymph node invasion, metastasis, poor differentiation level, advanced pre-surgery TNM stage and H19 overexpression in tumors were significantly associated with tumor recurrence $(\mathrm{P}=0.036, \mathrm{P}=0.001$, $\mathrm{P}=<0.01, \mathrm{P}<0.01$ and $\mathrm{P}=0.002$, respectively). Advanced TNM stage and increased H19 expression were identified as independent risk factors for recurrence in multivariate analysis $(\mathrm{P}=0.023$ and $\mathrm{P}=0.031$, respectively; Table IIA). Metastasis, poor differentiation level, advanced pre-surgery TNM stage and increased H19 expression in tumors were significantly associated with decreased OS rates using Cox's univariate proportional hazard analysis $(\mathrm{P}<0.01, \mathrm{P}<0.01$, $\mathrm{P}<0.01$ and $\mathrm{P}=0.001$, respectively). Increased H19 expression and advanced TNM stage were identified using Cox's multivariate analysis as independent risk factors for decreased OS rates $(\mathrm{P}=0.014$ and $\mathrm{P}=0.007$, respectively; Table IIB). These results suggested that overexpression of H19 is associated with unfavorable prognosis in patients with colorectal cancer.

H19 increases proliferation and migration of colon cancer cells. The expression level of H19 in cells transfected with the H19-expressing plasmid is significantly increased, compared with cells transfected with the control plasmid (Fig. 3A). MTT assays suggested that colon cancer cells transfected with the H19-expressing plasmid exhibited significantly increased viability potential (Fig. 3B). In addition, migration assays suggested that $\mathrm{H} 19$ overexpression significantly increased the migration potential of HCT-116 and SW-480 cells (Fig. 3C).

H19 induces EMT in colon cancer cells. Since decreased expression of E-cadherin and increased expression of vimentin- and EMT-associated transcription factors, including Snail, are well-known markers of the EMT process, the expression level of E-cadherin, vimentin and Snail was determined. H19 overexpression markedly decreased the expression of E-cadherin and increased the expression of vimentin and Snail, determined by western blot analysis (Fig. 3D). These results suggested that $\mathrm{H} 19$ overexpression induced the EMT process in colon cancer cells.

$H 19$ induces increased expression of F-actin in SW-480 cells. Previous studies identified that increased expression of F-actin is associated with increased migratory potential of cancer cells and is considered as a marker of EMT process, which prompted the determination of the expression of F-actin in SW-480 cells using immunofluorescence in the present study. The results suggested that $\mathrm{H} 19$ overexpression induced increased expression of F-actin and the increased expression was primarily located at the cell edge (Fig. 4).

\section{Discussion}

Although the mortality rate of colorectal cancer has decreased slightly in the last decade, it remains a life-threatening disease. The identification of novel factors involved in the development of colorectal cancer as novel therapeutic targets is required. H19, an imprinted and maternally expressed IncRNA, is abundant in embryonic tissues of endodermal and mesodermal origin, but is thought to be repressed following birth in the majority of tissues $(8,9)$. In addition, the expression level of $\mathrm{H} 19$ has been investigated in a number of types of cancer. Song et al (20) identified that there was an $>8$-fold increase in H19 expression in gastric tumor 
Table II. Cox's proportional hazard analysis.

A, Cox's proportional hazard analysis of recurrence-free survival

\begin{tabular}{|c|c|c|c|c|c|c|}
\hline \multirow[b]{2}{*}{ Variable } & \multicolumn{3}{|c|}{ Univariate } & \multicolumn{3}{|c|}{ Multivariate } \\
\hline & HR & $95 \% \mathrm{CI}$ & P-value & HR & $95 \% \mathrm{CI}$ & P-value \\
\hline Sex, female & 1.163 & $0.581-2.329$ & 0.670 & & & \\
\hline Age, $>60$ years & 1.379 & $0.665-2.862$ & 0.388 & & & \\
\hline T-stage, $3 / 4$ & 2.125 & $0.647-6.977$ & 0.214 & & & \\
\hline N-stage, 2 & 2.461 & $1.062-5.705$ & 0.036 & 1.311 & $0.503-3.418$ & 0.580 \\
\hline M-stage, 1 & 3.464 & $1.704-7.041$ & 0.001 & 1.471 & $0.621-3.487$ & 0.380 \\
\hline Tumor size, $>5 \mathrm{~cm}$ & 1.007 & $0.435-2.329$ & 0.987 & & & \\
\hline Differentiation, poor & 5.416 & $2.583-11.356$ & 0.000 & 1.667 & $0.670-4.418$ & 0.272 \\
\hline Mucin, present & 2.756 & $0.658-11.535$ & 0.165 & & & \\
\hline TNM-stage, III/IV & 5.223 & $2.339-11.666$ & 0.000 & 3.138 & $1.171-8.410$ & 0.023 \\
\hline H19 expression, high & 4.514 & $1.737-11.733$ & 0.002 & 3.037 & $1.107-8.329$ & 0.031 \\
\hline
\end{tabular}

B, Cox's proportional hazard analysis of overall survival rate

\begin{tabular}{|c|c|c|c|c|c|c|}
\hline \multirow[b]{2}{*}{ Variable } & \multicolumn{3}{|c|}{ Univariate } & \multicolumn{3}{|c|}{ Multivariate } \\
\hline & HR & $95 \% \mathrm{CI}$ & P-value & HR & $95 \%$ CI & P-value \\
\hline Sex, female & 0.722 & $0.353-1.476$ & 0.372 & & & \\
\hline Age, $>60$ years & 1.486 & $0.695-3.176$ & 0.307 & & & \\
\hline T-stage, $3 / 4$ & 3.089 & $0.736-12.973$ & 0.123 & & & \\
\hline $\mathrm{N}$-stage, 2 & 1.960 & $0.800-4.800$ & 0.141 & & & \\
\hline M-stage, 1 & 4.096 & $1.960-8.395$ & 0.000 & 1.127 & $0.463-2.743$ & 0.792 \\
\hline Tumor size, $>5 \mathrm{~cm}$ & 1.105 & $0.474-2.574$ & 0.818 & & & \\
\hline Differentiation, poor & 5.772 & $2.702-12.333$ & 0.000 & 2.261 & $0.950-5.378$ & 0.065 \\
\hline Mucin, present & 2.584 & $0.615-10.848$ & 0.195 & & & \\
\hline TNM-stage, III/IV & 5.570 & $2.384-13.016$ & 0.000 & 3.841 & $1.454-10.146$ & 0.007 \\
\hline H19 expression, high & 7.64 & $2.315-25.216$ & 0.001 & 4.028 & $1.332-12.183$ & 0.014 \\
\hline
\end{tabular}

P-values in italic indicate statistically significant differences. HR, hazard ratio; CI, confidence interval; TNM, tumor-node-metastasis; H19, long non-coding RNA-imprinted maternally expressed transcript.

tissues, compared with paired normal tissues. Furthermore, increased expression of H19 was markedly associated with an early recurrence of bladder cancer and may be considered a predictive marker for poor prognosis (14). Zhou et al (21) revealed that plasma $\mathrm{H} 19$ expression enabled the differentiation of early stage gastric cancer from controls with an area under the curve of 0.877 , sensitivity of $85.5 \%$ and specificity of $80.1 \%$ (18).

However, the underlying molecular mechanisms of the oncogenic function of $\mathrm{H} 19$ are complex. Previous studies have identified that $\mathrm{H} 19$ is able to regulate the expression of a number of types of cancer-associated protein, including calneuron 1 , the tumor suppressor retinoblastoma and ubiquitin ligase E3 family, by acting as the precursor of microRNA (miR)-675 (15,22,23). Additionally, a previous study suggested that H19 may function as a competing endogenous RNA for miR-138 and miR-200a, antagonizing their functions and thus leading to the derepression of their endogenous targets vimentin, zinc finger E-box-binding homeobox (ZEB) OL-9705-NPS 1 and ZEB2, all of which were associated with the EMT process (24). Further studies are required to determine the underlying molecular mechanisms of the role of H19 in distinct types of cancer.

In the present study, the expression level of H19 was identified to be markedly increased in colorectal tumor tissues, compared with adjacent normal tissues, which suggested that $\mathrm{H} 19$ serves a function in the tumorigenesis of colorectal cancer. Subsequently, the association between the expression level of H19 and the clinicopathological variables and patient survival was analyzed. Overexpression of H19 was identified to be associated with pre-treatment metastasis, poor differentiation level and advanced TNM stages. In addition, overexpression of $\mathrm{H} 19$ was revealed to be an independent risk factor for decreased RFS and OS. These results were consistent with previous studies, which suggests that H19 exhibits a tumorigenesis function in the development of a number of 
A

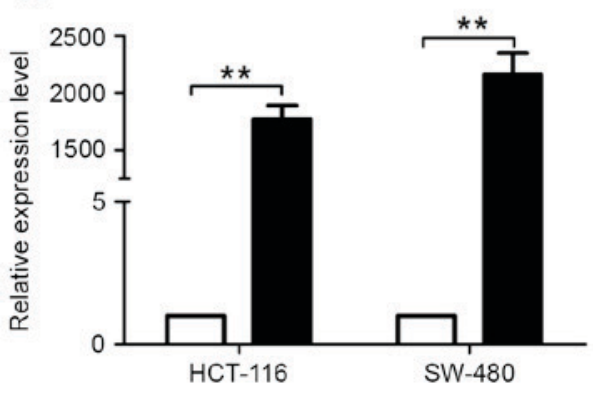

C

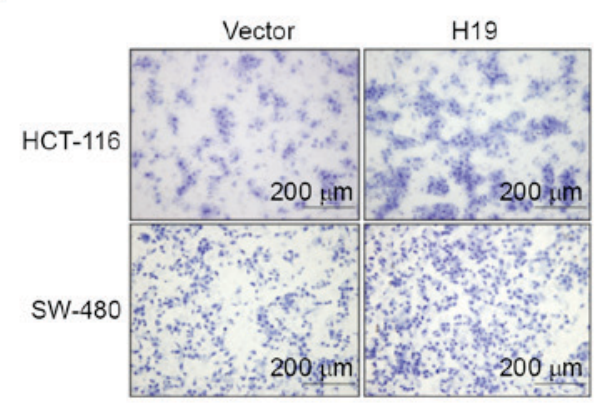

D
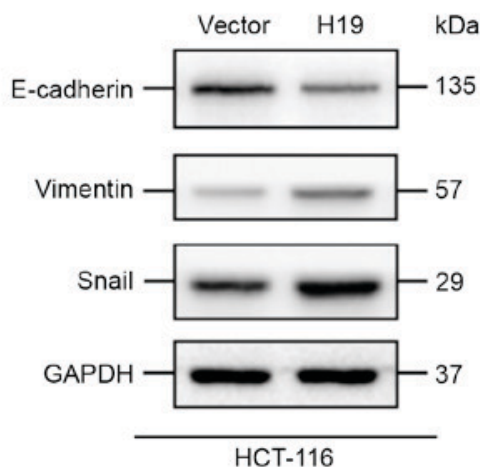

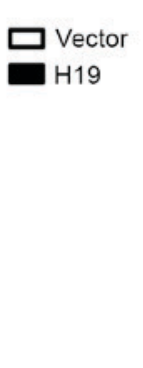

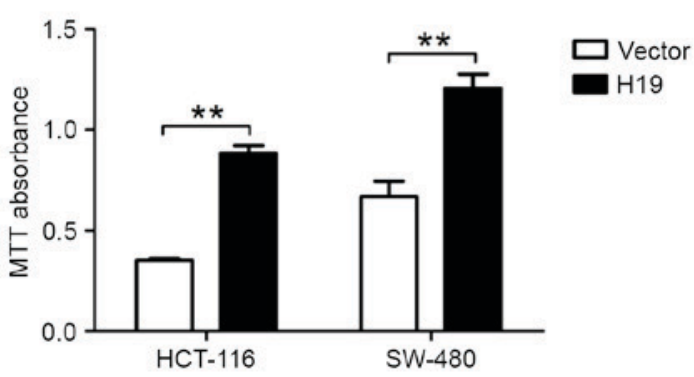
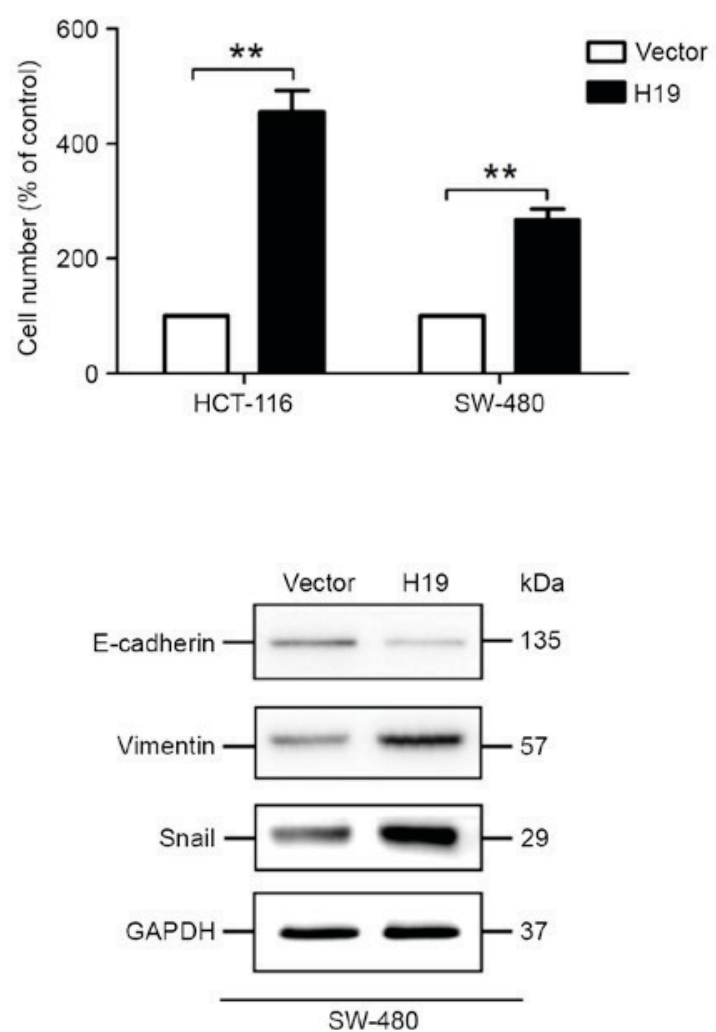

Figure 3. Effect of H19 overexpression on viability, migration and expression level of endothelial-mesenchymal transition-associated proteins. (A) Expression level of H19 determined using the quantitative polymerase chain reaction 3 days after transfection with H19-expressing plasmid or control plasmid, in HCT-116 and SW-480 cells. Cells transfected with H19-expressing plasmid exhibited a markedly increased expression level of H19, compared with cells transfected with the control plasmid (vector). (B) Cells were transfected with the H19 expressing plasmid or the control plasmid for $24 \mathrm{~h}$ and incubated for 5 days, followed by MTT assays. The viability level of cells overexpressing H19 is markedly increased, compared with cells transfected with the control plasmid (vector). (C) Migratory capacity of cells overexpressing H19 was markedly increased compared with cells transfected with the control plasmid (vector). (D) Expression levels of E-cadherin, vimentin and Snail were determined in HCT-116 and SW-480 cells $72 \mathrm{~h}$ after transfection with H19 or control plasmid. All experiments were performed in triplicate and repeated 3 times. Results are presented as the mean \pm standard error of the mean $(n=3)\left({ }^{* *} \mathrm{P}<0.01\right.$ vs. control). GAPDH was used as a loading control. H19, long non-coding RNA-imprinted maternally expressed transcript; E-cadherin, epithelial cadherin.

types of cancer (12-15). Notably, overexpression of H19 was identified to be associated with pre-treatment metastasis in the present study. Considering the important role of metastasis in the development of cancer, the effect of H19 on the metastasis potential of colon cancer cells, and the underlying molecular mechanism, was investigated.

Previous studies have suggested that H19 may be involved in the EMT process in a number of types of cancer cells $(25,26)$. EMT is considered to serve an important function in the process of metastasis and is characterized by 'cadherin switch', increased expression of vimentin and relevant transcription factors, including Snail $(27,28)$. In the present study, the expression levels of E-cadherin, vimentin and Snail were determined in HCT-116 and SW-480 cells. The results suggested that $\mathrm{H} 19$ overexpression markedly decreased the expression of E-cadherin, and increased the expression of vimentin and Snail, indicating that H19 overexpression induced the EMT process in colon cancer cells.

Since increased expression of F-actin is associated with increased migration potential of cancer cells and considered a marker of EMT process (29), the expression level of F-actin in SW-480 cells was determined using immunofluorescence. The 


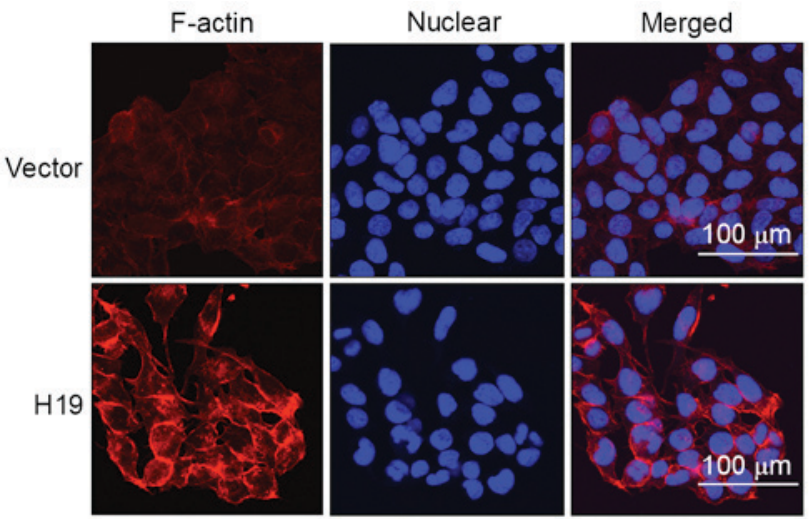

Figure 4. Expression of F-actin was increased in cells transfected with H19-expressing plasmid, compared with cells transfected with the control plasmid (vector). SW-480 cells cultured on slides were transfected with H19-expressing plasmid or control plasmid for $72 \mathrm{~h}$ before cells were fixed, and immunofluorescence of F-actin and nucleus was performed. F-actin, filamentous actin; H19, long non-coding RNA-imprinted maternally expressed transcript.

results suggested that $\mathrm{H} 19$ overexpression induced increased expression of F-actin and this increased expression was primarily located at cell edges.

The expression level of H19 is markedly increased in colorectal tumor tissues, compared with adjacent normal tissues, and overexpression of H19 is an independent risk factor for decreased RFS and OS rates in patients with colorectal cancer. Overexpression of H19 is additionally associated with increased viability and migration of colon cancer cells. In addition, it is hypothesized that the induction of the EMT process may be one of the underlying molecular mechanisms of the effect of $\mathrm{H} 19$ on the metastatic potential of colon cancer cells.

\section{Acknowledgements}

The authors thank Professor Ding-fang Bu for his technical support and recommendations with the present study, and all the staff of the Division of General Surgery (Peking University First Hospital, Beijing, China) for their assistance with the collection of samples and in the process of follow-up.

\section{References}

1. Siegel R, Ma J, Zou Z and Jemal A: Cancer statistics, 2014. CA Cancer J Clin 64: 9-29, 2014.

2. Chen W, Zheng R, Zeng $\mathrm{H}$ and Zhang S: The updated incidences and mortalities of major cancers in China, 2011. Chin J Cancer 34: 502-507, 2015.

3. Fearnhead NS, Wilding JL and Bodmer WF: Genetics of colorectal cancer: Hereditary aspects and overview of colorectal tumorigenesis. Br Med Bull 64: 27-43, 2002.

4. Wolpin BM and Mayer RJ: Systemic treatment of colorectal cancer. Gastroenterology 134: 1296-1310, 2008.

5. Ponting CP, Oliver PL and Reik W: Evolution and functions of long noncoding RNAs. Cell 136: 629-641, 2009.

6. Whitehead J, Pandey GK and Kanduri C: Regulation of the mammalian epigenome by long noncoding RNAs. Biochim Biophys Acta 1790: 936-947, 2009.

7. Li L, Feng T, Lian Y, Zhang G, Garen A and Song X: Role of human noncoding RNAs in the control of tumorigenesis. Proc Natl Acad Sci 106: 12956-12961, 2009.

8. Bartolomei MS, Zemel S and Tilghman SM: Parental imprinting of the mouse H19 gene. Nature 351: 153-155, 1991.
9. Tabano S, Colapietro P, Cetin I, Grati FR, Zanutto S, Mandò C, Antonazzo P, Pileri P, Rossella F, Larizza L, et al: Epigenetic modulation of the IGF2/H19 imprinted domain in human embryonic and extra-embryonic compartments and its possible role in fetal growth restriction. Epigenetics 5: 313-324, 2010.

10. Poirier F, Chan CT, Timmons PM, Robertson EJ, Evans MJ and Rigby PW: The murine H19 gene is activated during embryonic stem cell differentiation in vitro and at the time of implantation in the developing embryo. Development 113: 1105-1114, 1991.

11. Byun HM, Wong HL, Birnstein EA, Wolff EM, Liang G and Yang AS: Examination of IGF2 and H19 loss of imprinting in bladder cancer. Cancer Res 67: 10753-10758, 2007.

12. Berteaux N, Lottin S, Monté D, Pinte S, Quatannens B, Coll J, Hondermarck H, Curgy JJ, Dugimont T and Adriaenssens E: H19 mRNA-like noncoding RNA promotes breast cancer cell proliferation through positive control by E2F1. J Biol Chem 280: 29625-29636, 2005.

13. Kim SJ, Park SE, Lee C, Lee SY, Jo JH, Kim JM and Oh YK: Alterations in promoter usage and expression levels of insulin-like growth factor-II and H19 genes in cervical carcinoma exhibiting biallelic expression of IGF-II. Biochim Biophys Acta 1586: 307-315, 2002.

14. Ariel I, Sughayer M, Fellig Y, Pizov G, Ayesh S, Podeh D, Libdeh BA, Levy C, Birman T and Tykocinski ML: The imprinted $\mathrm{H} 19$ gene is a marker of early recurrence in human bladder carcinoma. Mol Pathol 53: 320-323, 2000.

15. Li H, Yu B, Li J, Su L, Yan M, Zhu Z and Liu B: Overexpression of lncRNA H19 enhances carcinogenesis and metastasis of gastric cancer. Oncotarget 5: 2318-2329, 2014.

16. Edge SB and Compton CC: The American Joint Committee on Cancer: The 7th edition of the AJCC cancer staging manual and the future of TNM. Ann Surg Oncol 17: 1471-1474, 2010.

17. Livak KJ and Schmittgen TD: Analysis of relative gene expression data using real-time quantitative PCR and the 2(-Delta Delta C(T)) method. Methods 25: 402-408, 2001.

18. Kiernan JA: Histological and Histochemical Methods: Theory and Practice. 4th edition. Bloxham, UK: Scion, 2008.

19. Chen S, Zhu J, Zuo S, Ma J, Zhang J, Chen G, Wang X, Pan Y, Liu Y and Wang P: 1,25(OH)2D3 attenuates TGF- $\beta 1 / \beta 2$-induced increased migration and invasion via inhibiting epithelial-mesenchymal transition in colon cancer cells. Biochem Biophys Res Commun 468: 130-135, 2015.

20. Song H, Sun W, Ye G, Ding X, Liu Z, Zhang S, Xia T, Xiao B, $\mathrm{Xi} Y$ and Guo J: Long non-coding RNA expression profile in human gastric cancer and its clinical significances. J Transl Med 11: 225, 2013.

21. Zhou X, Yin C, Dang Y, Ye F and Zhang G: Identification of the long non-coding RNA H19 in plasma as a novel biomarker for diagnosis of gastric cancer. Sci Rep 5: 11516, 2015.

22. Tsang WP, Ng EK, Ng SS, Jin H, Yu J, Sung JJ and Kwok TT: Oncofetal H19-derived miR-675 regulates tumor suppressor RB in human colorectal cancer. Carcinogenesis 31: 350-358, 2010.

23. Vennin C, Spruyt N, Dahmani F, Julien S, Bertucci F, Finetti P, Chassat T, Bourette RP, Le Bourhis X and Adriaenssens E: H19 non coding RNA-derived miR-675 enhances tumorigenesis and metastasis of breast cancer cells by downregulating c-Cbl and Cbl-b. Oncotarget 6: 29209-29223, 2015.

24. Liang WC, Fu WM, Wong CW, Wang Y, Wang WM, Hu GX, Zhang L, Xiao LJ, Wan DC, Zhang JF and Waye MM: The lncRNA H19 promotes epithelial to mesenchymal transition by functioning as miRNA sponges in colorectal cancer. Oncotarget 6: 22513-22525, 2015.

25. Ma C, Nong K, Zhu H, Wang W, Huang X, Yuan Z and Ai K: H19 promotes pancreatic cancer metastasis by derepressing let-7's suppression on its target HMGA2-mediated EMT. Tumour Biol 35: 9163-9169, 2014.

26. Matouk IJ, Raveh E, Abu-lail R, Mezan S, Gilon M, Gershtain E, Birman T, Gallula J, Schneider T, Barkali M, et al: Oncofetal H19 RNA promotes tumor metastasis. Biochim Biophys Acta 1843: 1414-1426, 2014.

27. Tsanou E, Peschos D, Batistatou A, Charalabopoulos A and Charalabopoulos K: The E-cadherin adhesion molecule and colorectal cancer. A global literature approach. Anticancer Res 28: 3815-3826, 2008.

28. Costa LC, Leite CF, Cardoso SV, Loyola AM, Faria PR, Souza PE and Horta MC: Expression of epithelial-mesenchymal transition markers at the invasive front of oral squamous cell carcinoma. J Appl Oral Sci 23: 169-178, 2015.

29. Ouderkirk JL and Krendel M: Non-muscle myosins in tumor progression, cancer cell invasion and metastasis. Cytoskeleton (Hoboken) 71: 447-463, 2014. 